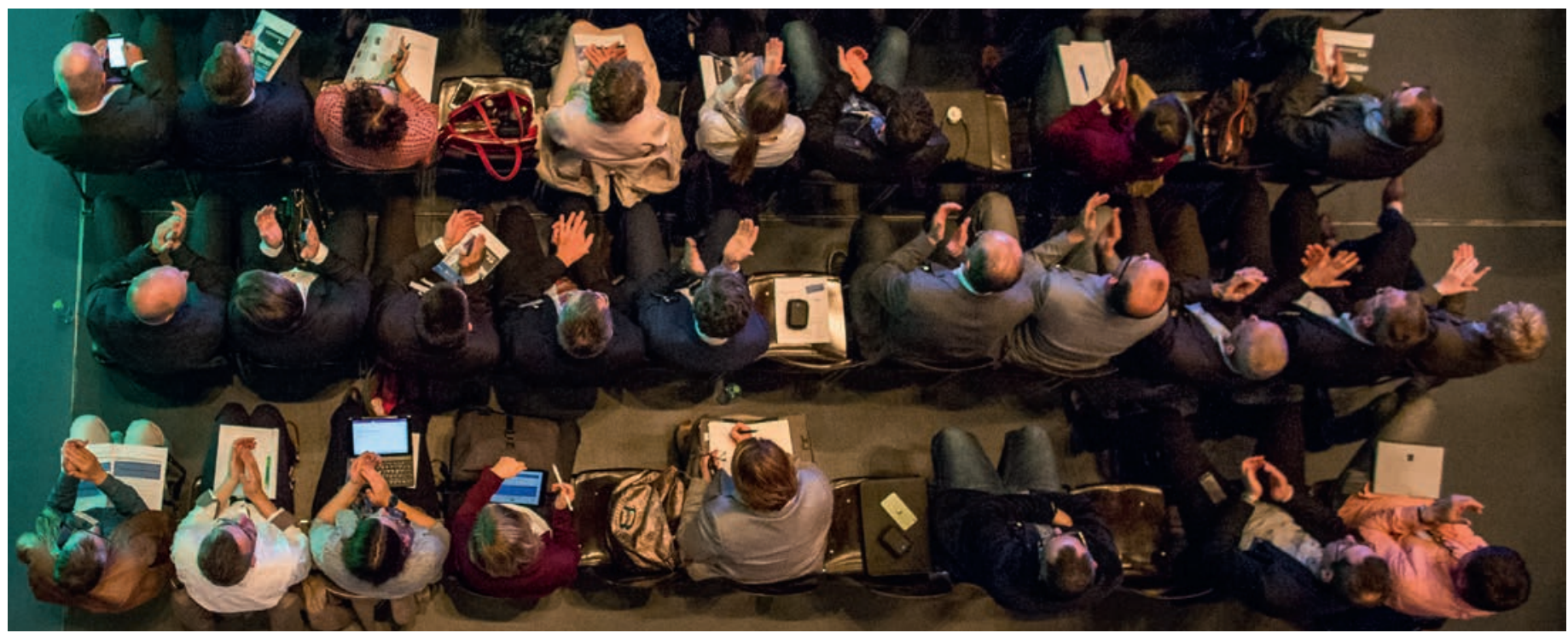

Luzerner Trendtage Gesundheit 2017

\title{
Digital unterstützte Körperoptimierung
}

\section{Anna Sax}

Lic. oec. publ., MHA, Mitglied der Redaktion

Wer Ende März die Luzerner Trendtage Gesundheit besuchte, erhielt einen Eindruck davon, was Big Data und digitale Transformation in Zukunft für uns bereithalten. Die Erwartungen sind immens. Die Frage aber, welchen Nutzen all die Daten, die wir in die Rechner füttern, für unsere Gesundheit bringen, bleibt offen. Die Ärztezeitung sammelte Eindrücke vom Kongress.

\section{Résumé}

Cette année, les Trendtage Gesundheit Luzern avaient pour thème la «Santé 4.0». Les visiteuses et visiteurs ont pu y découvrir des aperçus de l'avenir numérique de l'industrie de la santé. Les conférences portaient sur le big data, les diagnostics basés sur des algorithmes, les interventions assistées par ordinateur, les applications destinées à optimiser le comportement de santé, les technologies portables (wearables) pour l'optimisation du corps et de nombreuses autres innovations du secteur informatique. II reste de nombreuses questions en suspens. Réussirons-nous par exemple à garder une vue d'ensemble des quantités énormes et croissantes de données et à les exploiter judicieusement dans la santé? Comment les applis, wearables et autres gadgets en vogue influenceront-ils notre approche de la santé et de la maladie? La plupart des intervenants considèrent le big data comme un atout pour la science et certains attendent de la population qu'elle mette ses données de santé à disposition pour le bien de la communauté.
Das Sentinella-Meldesystem ist von gestern. Was sind schon ein paar Tausend Meldungen und Laborwerte im Vergleich zu Millionen und Abermillionen von verschnupften Menschen weltweit, die ihre Symptome in die Suchmaschine eingeben und damit Google zu einem riesigen Datenschatz verhelfen? Google sah das Potenzial der Gesundheitsdaten und baute einen Dienst auf mit Informationen über die Entwicklung von Grippe-Epidemien. «Google Flu Trends» war zunächst gratis verfügbar, das freute die Forschungswelt. Doch das Glück dauerte nicht lange, denn die Gesundheitsdaten, von Internet-Nutzerinnen und -Nutzern bereitwillig preisgegeben, lassen sich zu Geld machen. So viel Geld, dass es für die Forschenden des Digital Epidemiology Lab an der EPFL in Lausanne zu einem Problem wurde. Sie konnten und wollten sich die teuren Google-Dienste nicht länger leisten. Also nahmen 


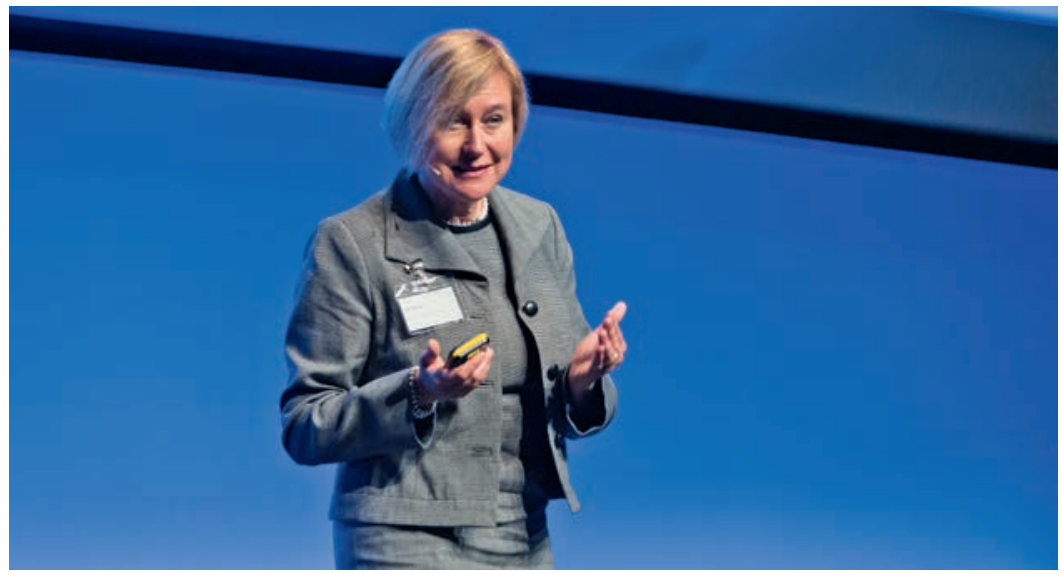

Ines Bersch-Porada, Expertin für Exoskelette, erwartet keine raschen Wunder.

sie einen Umweg, wie EPFL-Professor Marcel Salathé nicht ohne Stolz in seinem Referat erläuterte: Die meisten Google-Links führen nämlich zu Wikipedia, und das wiederum ist öffentlich zugänglich. So können die Forschenden diverse Krankheiten "tracken" und zum Beispiel auch Nebenwirkungen von Medikamenten erfassen. Twitter hat ebenfalls einiges an Datenmaterial zu bieten, denn die Leute reden gerne über ihre Gebresten und teilen der Welt über das Smartphone ihre Meinung zu allem Möglichen mit. Zum Beispiel zwitschern sie, was sie von Impfungen halten, und schon verfügen die digitalen Epidemiologen über Informationen zur Akzeptanz von Impfungen in ausgewählten Ländern.

\section{Zählt eure Schritte!}

Algorithmen sind in der Lage, Bilder von Hautveränderungen zu analysieren, und zwar mindestens so gut wie Menschen oder sogar besser, wie Marcel Salathé weiter ausführte. Dies, nachdem sie mit 130000 Bildern gefüttert wurden, die Dermatologen zuvor beurteilt hatten. Die Software «Deep learning», die maschinelle Diagnosen und noch viel mehr möglich macht, steht gratis zur Verfügung, und die Forschenden bedienen sich gerne. Sie werten unsere Gesundheitssorgen aus, die wir bereitwillig den Clouds anvertrauen, und zwar zum Nutzen der Allgemeinheit. Davon ist zumindest Marcel Salathé so sehr überzeugt, dass er sagte: «Es ist unsere zivile und wissenschaftliche Pflicht, dafür zu sorgen, dass diese Daten für das öffentliche Wohl benutzt werden können». Wir sollen also nebst unseren Organen auch unsere Daten spenden. Womöglich sollten wir einen (digitalen) Datenspende-Ausweis auf uns tragen, wie der Wearables-Spezialist Christian Stammel vorschlug.

Zählt eure Schritte! Fotografiert eure Teller! Schickt eure Blutdruck- und Blutzuckerwerte in die Cloud! Solche Aufforderungen waren in Luzern allgegenwärtig. Die Vertreter von Industrie, Beratungsfirmen und
Kommunikationsunternehmen überboten sich gegenseitig mit Ideen, was man alles mit digitalen Daten anstellen könnte. Christian Stammel zeigte sich fasziniert von «allem, was man am Körper, in Körpernähe oder auch im Körper tragen kann», solange es verwertbare Informationen aussendet. Nein, Wearables sind normalerweise keine Kleider. Es können Pulsmesser sein, oder auch sogenannte «Smart Pills», die beim Auflösen im Körper einen elektrischen Impuls aussenden und die Aussenwelt darüber informieren, dass sie am Ziel angekommen sind.

\section{«Wir sind alle Ignoranten»}

Die allermeisten Referenten (30 von 33 waren männlich) waren sich darüber einig, dass Daten, die aus Wearables oder aus der Verwendung von Internet und Smartphones hervorgehen, die Wissenschaft weiterbringen. Mehrere betonten, die Daten dürften, ja müssten sogar für die Wissenschaft und für den Gesundheitsmarkt nutzbar gemacht werden. Nur Einzelne zeigen sich skeptisch gegenüber der grenzenlosen Begeisterung für Big Data, zum Beispiel Professor Christian Lovis, Direktor der Division of Medical Information Sciences am Genfer Universitätsspital HUG. Er merkte in seinem Referat leicht sarkastisch an, dass Maschinen, die künstliche Intelligenz erzeugen sollten, von lauter Ignoranten gefüttert würden: «Es erscheinen so viele Studien, und wir können höchstens ein Tausendstel davon lesen. Was ist nun wichtig? Was ist evident?» Die Wissenschaft funktioniere eben ähnlich wie eine direkte Demokratie, wo die Mehrheit darüber entscheide, was wahr sei. Nicht erst seit Donald Trump wüssten wir, so Christian Lovis, dass es auch eine rhetorische Wahrheit gibt. Die Maschinen lernten aufgrund existierender Erkenntnisse der Menschheit, die sich oftmals als Dogmen herausstellten. Zum Beispiel die oben erwähnten «Google flu trends»: Der Dienst habe die Grippe zwar schön vorausgesagt, viel schneller als das Sentinella Netzwerk, aber wie sich später herausgestellt habe, völlig falsch. «Wenn künstliche Intelligenz die schönste Frau wählen muss, dann ist diese weiss, jung, blond und trägt wenig Kleider», verrät Christian Lovis. Der Computer ist eben doch relativ einfach gestrickt und gibt nur das wieder, was ihm zuvor eingefüttert wurde.

\section{Chat gegen Übergewicht}

Keine «Smart Pills», aber «Digitale Pillen» entwickelt Tobias Kowatsch vom Center for Digital Health Interventions der Universität St. Gallen und der ETH Zürich. Er arbeitet an digitalen Interventionen, die helfen sollen, Gesundheitsverhalten zu unterstützen. Im Gespräch 


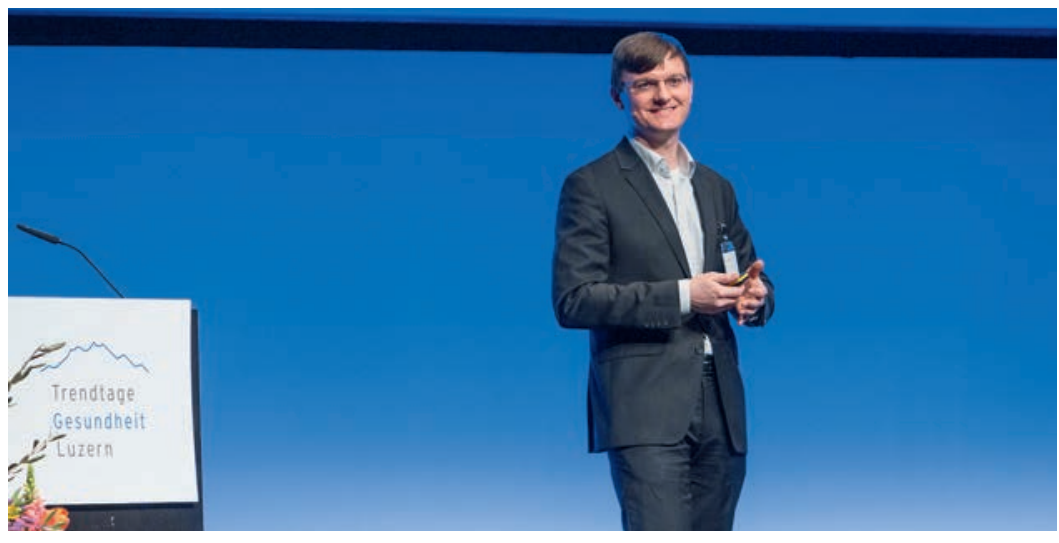

Tobias Kowatsch will durch digitale Interventionen einen gesunden Lebensstil fördern.

mit der Ärztezeitung betont er sein Interesse für die Schnittstelle zwischen Informatik, Psychologie und Medizin. Das von einem Krankenversicherer unter viel medialer Beachtung eingeführte Programm, das Schritte zählt und körperliche Aktivität belohnt, sei vor allem interessant für diejenigen, «die sich mehr im Alltag bewegen möchten und eine Alternative zum Fitnesszentrum suchen", erklärt Tobias Kowatsch. "Die grosse Herausforderung besteht allerdings darin, Leute in ihrem Gesundheitsverhalten zu unterstützen, die bedingt durch Krankheitsbilder wie Depression, Diabetes oder starkes Übergewicht nur sehr schwer zu motivieren sind.» So wurden im Rahmen des SNF Projekts PathMate2 von Kinderärzten und Informatikern die digitalen Coaches «Anna \& Lukas» entwickelt, welche interaktiv über Textnachrichten und Sensordaten des Smartphones übergewichtige Kinder coachen. Die Kinder erhalten täglich Aufgaben in den Bereichen Bewegung, Ernährung und Stressmanagement und werden bei ihrer Erfüllung belohnt. Die täglichen Aufgaben, so Tobias Kowatsch, würden nach den ersten beiden Monaten zu ca. $60 \%$ erfolgreich absolviert.

\section{Roboter der besonderen Art}

Ein viel beachtetes Thema der Luzerner Trendtage waren die Exoskelette. Diese sollen, so die Hoffnung vieler Betroffener und Therapeutinnen, Querschnittgelähmten irgendwann das Gehen ermöglichen. Expertin für diese besonderen Roboter ist Ines BerschPorada, die als Physiotherapeutin seit vielen Jahren im Schweizer Paraplegikerzentrum in Nottwil tätig ist. Sie ist unter anderem verantwortlich für die Funktionelle Elektrostimulation und den Einsatz von Robotic. Ines Bersch ist realistisch: «Exoskelette sind kein Ersatz für einen Rollstuhl», stellt sie im Gespräch mit der Ärztezeitung klar. Für die erfahrene Physiotherapeutin liegt der Wert der Exoskelette im Therapiebereich. Sie können beispielsweise dabei helfen, das Gleichgewicht zu verbessern. Und sie werden eingesetzt zur Unterstützung der Tonus-Regulation oder der Schmerztherapie. Die elektronisch gesteuerten Schienensysteme können auch die Rehabilitation bei inkompletten Lähmungen unterstützen. Noch ist aber die Evidenzlage dünn: Studien, die unabhängig von Marktanbietern erfolgten, gebe es kaum, so Ines Bersch. Bis die Exoskelette alltagstauglich sein werden, wird es noch lange dauern. Die Hoffnung, dass diese besonderen Roboter eines Tages das Leben von Paraplegikerinnen und Tetraplegikern zumindest erleichtern werden, auch wenn sie nicht zu vollkommener Mobilität verhelfen, ist bei Ines Bersch trotz aller Vorbehalte zu spüren. Und sie will die Gesellschaft nicht aus ihrer Verantwortung entlassen: «Es muss weiter vorangehen mit dem Abbau von Hindernissen, die Menschen im Rollstuhl behindern.»

\section{Die Trendtage liegen im Trend}

Abgesehen von Ausnahmen wie den Vorträgen von Kowatsch und Bersch tauchte die Frage nach dem Nutzen für die Patientinnen und Patienten im Verlauf der beiden Kongresstage nur am Rand auf. Der Luzerner Gesundheitsdirektor Guido Graf stellte zwar in seiner Begrüssungsbotschaft im Programmheft eine Reihe von Fragen zum Gesundheitsnutzen der digitalen Transformation, doch kaum eine davon wurde beantwortet. Zwei Beispiele: Gelingt es, die riesigen und wachsenden Datenmengen immer besser zu überblicken und sie für medizinische Behandlungen sinnvoll zu nutzen? Wie verändern Apps, Wearables und andere trendige mobile Gadgets unseren Umgang mit Gesundheit und Krankheit? Die Luzerner Trendtage Gesundheit 2017 lieferten wenig neue Erkenntnisse zu solchen gesundheitsrelevanten Fragestellungen. Vielleicht ist es dazu einfach noch zu früh. Dass das Thema der digital unterstützten Körperoptimierung aber im Trend liegt, zeigt sich daran, dass am darauffolgenden Wochenende in der NZZ am Sonntag mehrere Beispiele für den Einsatz von Computern und Wearables im Spitzensport vorgestellt wurden: So versuchte etwa der Sportartikel-Konzern Nike die Marathon-Bestzeit unter zwei Stunden drücken, indem die Athleten mit «Smart Pills» und weiteren Gadgets ausgerüstet wurden, die es ihnen ermöglichen sollten, immer haarscharf an der Leistungsgrenze zu rennen. Der Versuch fand auf einer Formel-1-Rennstrecke statt, er scheiterte nur knapp. Hier wie in der Gesamtbetrachtung der Trendtage verschwimmen die Grenzen zwischen medizinischem Nutzen, Leistungssteigerung und purem Vergnügen am technischen Schnickschnack.

Bildnachweise

Bruno Näpflin / Monika Wittwer, Akzent Media GmbH 